\title{
Liver Ligament
}

National Cancer Institute

\section{Source}

National Cancer Institute. Liver Ligament. NCI Thesaurus. Code C32997.

Any of the ligaments associated with the liver. 